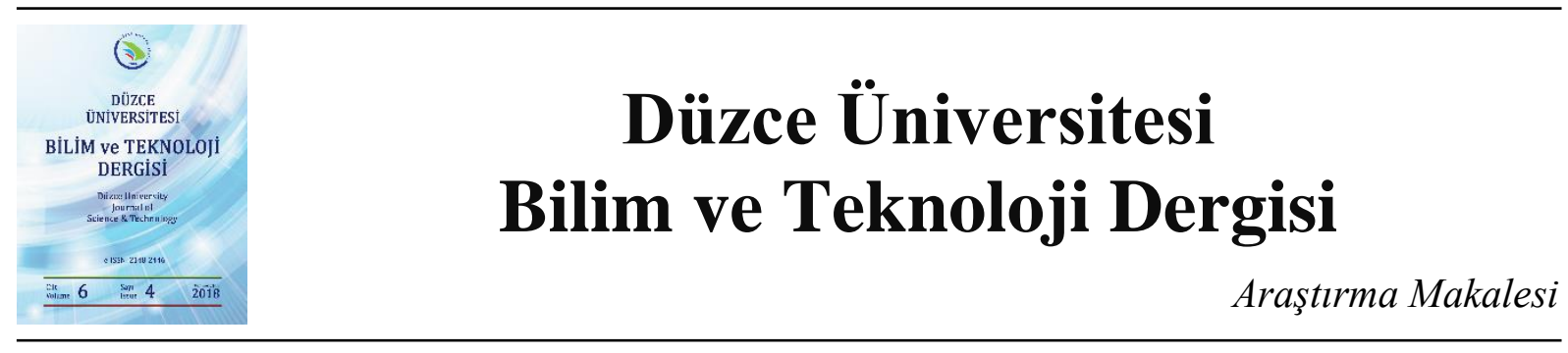

\section{Güç Dönüştürücülerinin Kapalı Döngü İçinde Benzetimi}

\author{
Fatih EVRAN ${ }^{\text {a,* }}$ \\ ${ }^{a}$ Elektrik-Elektronik Mühendisliği Bölümü, Mühendislik Fakültesi, Düzce Üniversitesi, Düzce, TÜRKIYE \\ * Sorumlu yazarin e-posta adresi: fatihevran@duzce.edu.tr
}

\begin{abstract}
ÖZET
Bu çalışmanın amacı, güç dönüştürücüleri için kapalı döngü içerisinde etkili bir benzetim ortamı oluşturmaktır. Kullanılan benzetim gerçek zamanlı olup, geleneksel benzetim yazılımlarından farklıdır. Gerçek zamanlı benzetimlerde yüksek hassasiyetli sonuçlar elde edebilmek için örnekleme zaman aralı̆̆ının oldukça küçük olması gerekmektedir. Bu ise sistemin maliyetinin artmasına yol açmaktadır. Bu çalışmada, açık kaynaklı bir işletim sisteminde, güç dönüştürücülerinde durum uzay ortalama yaklaşımını kullanarak gelişmiş mikrodenetleyiciler için çok düşük maliyetli gerçek zamanlı bir donanım test ortamının ayrıntılı bir uygulaması sunulmuştur. Örnek olarak, AA (Alternatif akım)-DA (Doğru akım) güç faktörü düzeltme dönüştürücüsünün denetleyicisi tasarımı için gerçek zamanlı bir benzetiminin test yaklaşımı uygulanmıştır. Önerilen gerçek zamanlı benzetimin geçerliliği MATLAB/ Simulink benzetim sonuçları ile karşılaştırılarak doğrulanmıştır.
\end{abstract}

Anahtar Kelimeler: Gerçek Zamanlı Benzetim, Kapalı Döngü İ̧erisinde Donanım, Güç Dönüştürücüsü, Gü̧̧ Faktörü

\section{Hardware in the Loop Simulation of Power Converters}

\author{
ABSTRACT
}

\begin{abstract}
The purpose of this study is to create an effective hardware in the loop simulation platform for power converters. The simulation used is real-time and is different from traditional simulation software. In order to obtain highprecision results in real-time simulations, the sampling time interval must be very small. This leads to an increase in the cost of the system. In this study, a detailed implementation of a very low cost real-time hardware test environment is presented by using state space averaging approach in power converters in an open source operating system for advanced microcontrollers. For example, the testing approach of a real-time simulation of the controller design of the AC-DC power factor correction converter has been implemented. The validity of the proposed real-time simulation is verified by comparing with simulation results in MATLAB/Simulink
\end{abstract}

Keywords: Real-Time Simulation, Hardware in the loop, Power Converter, Power Factor 


\section{GiRiș}

$\mathrm{M}$ ühendislik çalışmalarında benzetim tekniklerinden yararlanmak çok yaygın bir uygulamadır. Özellikle malzeme ve cihaz güvenliğinin önemli olduğu güç elektroniği uygulamalarında benzetim çalışmaları daha da büyük önem kazanmaktadır. Geleneksel olarak, Simulink, Pspice ve Labview gibi gerçek zamanlı olmayan benzetim araçları, güç elektroniği çalışmaları için geliştirme ve sorun çözmede kullanılmaktadır. Ancak bu tür yazılımların iki büyük olumsuzluğu vardır: Birincisi, bilgisayar işlemcisi bu benzetim araçlarında modellenmiş sistemle aynı hızda çalışmamaktadır. Yani, işlemci tarafından harcanacak zaman benzetim süresinden oldukça büyüktür. İkincisi, bu zaman farkı gerçek zamanlı olmayan benzetim aracına dışarıdan bir donanım bağlanmasını engellemektedir. Dolayısıyla, sistemlerin davranışlarını analiz edebilmek mümkün olmadığı için gerçek zamanlı benzetim araçlarına ihtiyaç duyulmaktadır. Kapalı döngü içerisinde donanım desteği ise modellenmiş bir sistemin gerçek donanım bileşenleri ile etkileşime girmesine izin veren bir tür gerçek zamanlı bir benzetim tekniğidir.

Gerçek zamanlı benzetim (GZB) ve donanım testinin genel yapısı Şekil 1'de gösterilmiştir. Şekil 1'de gösterildiği gibi GZB ortamının yapısında bulunan yazılım ve donanım kısımları yardımıyla, farklı gerçek çalışma yükleri ve koşulları altında sistemin bir donanımı test edilmektedir.

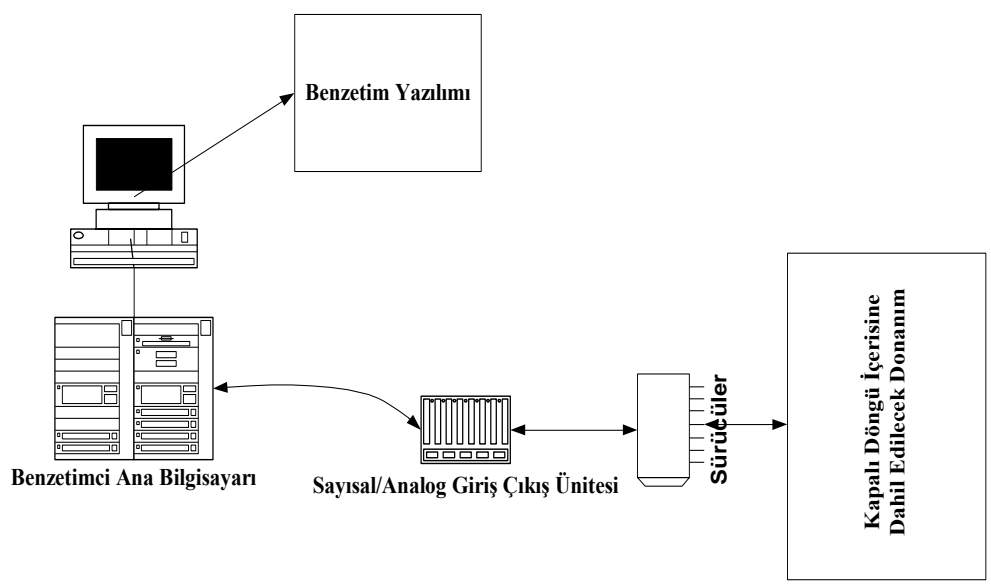

Şekil 1. Gerçek zamanlı benzetim ortamının genel yapısı.

GZB sistemlerinde işlemci üniteleri olarak genellikle mikroişlemciler, Alan Programlanabilir Kapı Dizileri (FPGA) ve Sayısal İşaret İşleyiciler (DSP) kullanılmaktadır. GZB sistemleri, havacılık [1- 3], otomotiv [4- 6], yenilenebilir enerji kaynakları [7- 9], iletim hattı [10] ve robotik [11] ve güç elektroniği [12- 17] gibi çeşitli alanlarda kullanılmaktadır. FPGA kullanarak örnekleme zaman aralığı küçülmesine rağmen güç elektroniği uygulamalarında kullanılan modelleme dilindeki kütüphane eksikliği sistem geliştirme süresini arttırmaktadır [12-14]. Bu sistemler sadece gerçek zamanlı olup, sistemlerde herhangi bir donanım testi yapılmamaktadır. Güç dönüştürücüleri uygulamalarında sistemi tasarlamak ve test edebilmek için sayısal denetleyici algoritmalarının geliştirilmesi gerekmektedir [16- 18]. Ancak burada kullanılan yazılım açık kaynaklı olmasına rağmen örnekleme zaman aralığı küçük değildir. GZB araçlarında donanım testi için uygun yüksek hızlı Giriş/Çıkış yuvalarına ihtiyaç duyulmaktadır. Ayrıca, geleneksel gerçek zamanlı bir benzetimin nispeten uzun bir benzetim örnekleme süresine sahip olmasından dolayı, yüksek anahtarlama frekansına sahip DA-DA dönüştürücülerinde donanım ya da sayısal denetleyici testini yapmak mümkün olmamaktadır. Dolayısıyla, seçilen örnekleme adım aralığı içinde bütün sistem denklemlerinin hesaplanması 
gerekmektedir. Bu çalışmada, GZB tekniğinin güç elektroniği sistemlerine uyarlanmasına çalış1lmıştır. $\mathrm{Bu}$ amaçla, kullanılan güç elektroniği topolojisi olan AA-DA güç faktörü düzeltme (GFD) dönüştürücüsünün DSP'de gömülü olan sayısal denetleyicisinin tepkisi kapalı döngü içerisinde donanım desteği ile izlenilmektedir. Modellenen sistem denklemlerinin, açık kaynaklı bir işletim sisteminde seçilen küçük zaman boyutu içinde yeterince hızlı çözülebilmesi için alternatif bir yöntem olarak durum uzay ortalama yöntemi kullanılmaktadır. Güç elektroniği bölümü ise kişisel bilgisayarda gerçek zamanlı olarak çalışmaktadır. Elde edilen benzetim sonuçları, sayısal denetleyici değişkenlerinin benzetim yazılımında kolaylıkla değiştirilebildiğini ve güç elektroniği sistemlerinin test edilmesinin hızlı olabileceğini göstermektedir. $\mathrm{Bu}$ çalışmanın içeriği aşağıdaki gibi düzenlenmiştir. Bölüm II önerilen gerçek zamanlı benzetim yapısını anlatmaktadır. Bölüm III önerilen dönüştürücü devresini açıklamaktadır. Bölüm IV ise önerilen dönüştürücünün etkinliğini doğrulamak için benzetim sonuçlarını vermektedir.

\section{II. ÖNERILEN GERÇEK ZAMANLI BENZETIM YAPISI}

GZB sistemini, 3 GHz'de çalışan dört çekirdekli Intel işlemciye sahip ve hafızası 16 GB RAM olan bir bilgisayar oluşturmaktadır. Verimli bir performans elde etmek için QNX Neutrino 6.2 adı verilen bir açık kaynak gerçek zamanlı işletim sistemi kullanılmaktadır. Geliştirilen kesme adım aralığı 9 mikrosaniye'dir. Ayrıca, donanım testini sağlayabilmek için bilgisayara yerleştirilmiş bir PCI-E Giriş /Çıkış yuvası bulunmaktadır. Sayısal denetleyici algoritmaları ise DSP'ye yüklenilmektedir. Önerilen GZB sisteminin yapısı Şekil 2'de gösterilmiştir.

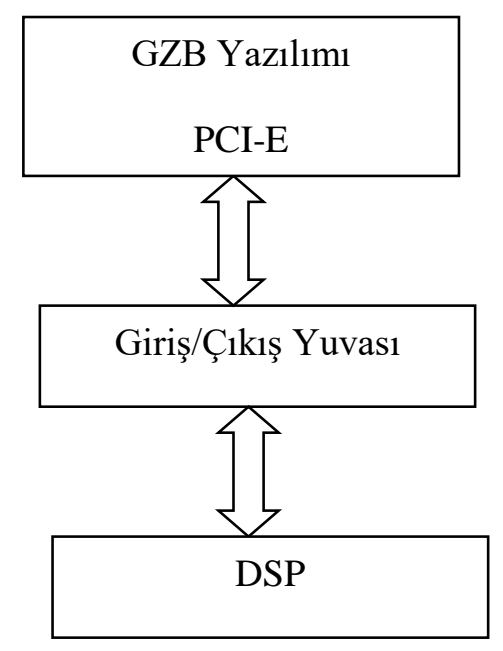

Şekil 2. Önerilen GZB sistem yapısı

\section{III. İNCELENEN GFD DEVRESI}

GFD dönüştürücülerinde kullanılan farklı topolojiler vardır. Bu çalışmada kullanılan topoloji Şekil 3'te gösterilmiştir. Bu topolojinin benzer GFD dönüştürücülere göre önemli bir kazanımı, yarı iletken anahtarların iletim kayıplarının daha düşük olmasıdır [19]. Girişte doğrultucu içermeyen bu topoloji, 
AA giriş geriliminden çıkışta DA gerilimini elde etmek için iki tane tek faz Yükselten (Boost) DA-DA dönüştürücüsünden meydana gelmektedir.

Giriş gerilimin pozitif ve negatif döngüsü sırasında sırasıyla $S_{l}$ ve $S_{2}$ anahtarlarının denetlenmesiyle giriş akımının sinüzoidal ve çıkış geriliminin sabit olması sağlanılmaktadır. Sürekli iletim kipinde çalışan dönüştürücünün giriş geriliminin pozitif ve negatif döngüsü sırasında oluşan farklı çalışma kipleri sırasıyla Şekil 4 (a) ve 4 (b) 'de gösterilmiştir.

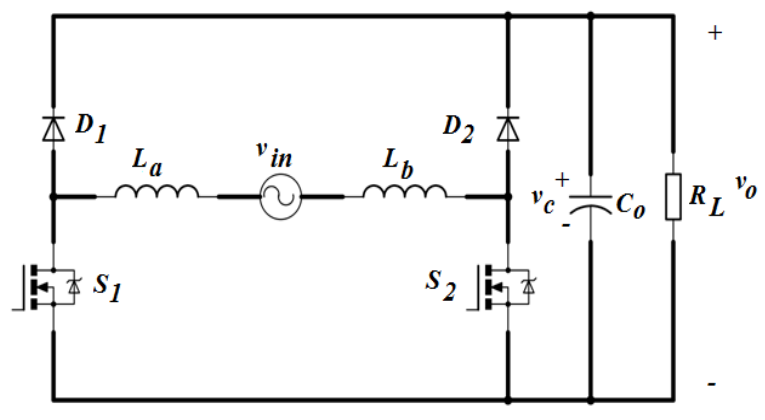

Şekil 3. Incelenen GFD devresi

(a)
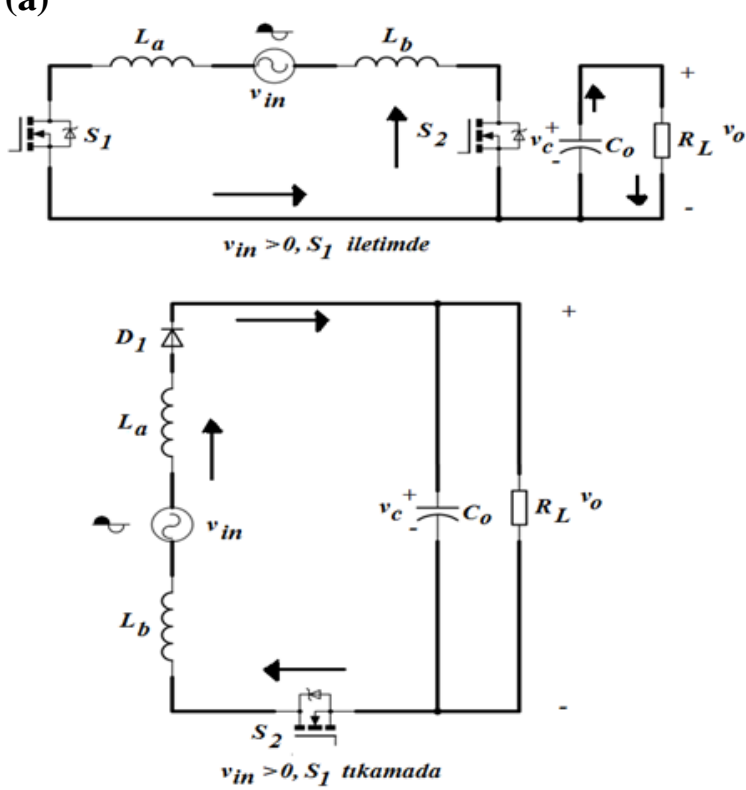

(b)
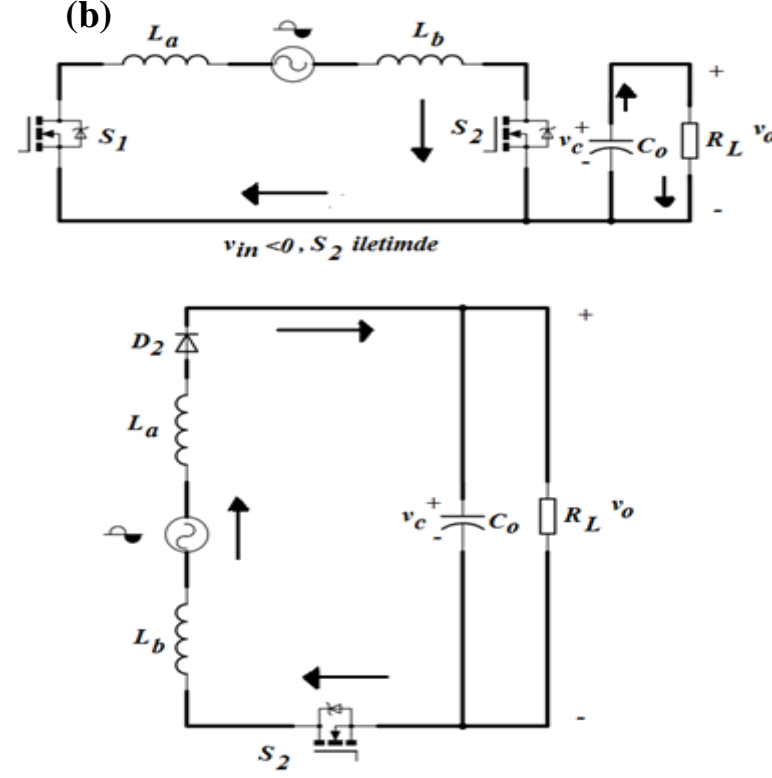

$v_{\text {in }}<0, S_{2}$ tikamada

Şekil 4. Anahtarlar iletimde ve tıkamada iken dönüş̧ürücünün eşdeğer devreleri a) Giriş gerilimi pozitif,(b) Giriş gerilimi negatif

Giriş gerilimi pozitif iken dönüştürücünün matematiksel modeli Eşt. 1 ve Eşt. 2'de verilmiştir. $S_{I}$ anahtarı iletimde iken kaynak akımı, $S_{l}$ anahtarı ve $S_{2}$ anahtarının eklem diyotu üzerinden akmaktadır. $\mathrm{Bu}$ süre zarfında endüktans enerji depolamaktadır ve kondansatör yük üzerinden enerjisini boşaltmaktadır $\left(0<t<d T_{s}\right)$. 
$v_{i n}=L \frac{d i_{L}}{d t}$

$C_{0} \frac{d v_{0}}{d t}=-\frac{v_{0}}{R_{L}}$

$S_{1}$ anahtarı tıkamada iken kaynak akımı, $S_{2}$ anahtarının eklem diyotu ile paralel bağlanmış olan kondansatör ve yük üzerinden akmaktadır $\left(d T_{s}<t<T_{s}\right)$.

$v_{i n}-v_{0}=L \frac{d i_{L}}{d t}$

$C_{0} \frac{d v_{0}}{d t}=i_{L}-\frac{v_{0}}{R_{L}}$

Giriş gerilimi negatif iken oluşan denklemler, giriş gerilimi pozitif iken oluşan denklemler ile aynıdır (Eşt.1-4). $0<t<d T_{s}$ ve $d T_{s}<t<T_{s}$ zaman aralıklarına karşılık gelen devre modellerin matrisleri sirasıyla Eşt. 5 ve Eşt. 6 'da verilmektedir.

$$
\begin{aligned}
& {\left[\begin{array}{c}
\frac{d\left|i_{L}\right|}{d t} \\
\frac{d v_{0}}{d t}
\end{array}\right]=\left[\begin{array}{cc}
0 & 0 \\
0 & -\frac{1}{R_{L} C_{0}}
\end{array}\right]\left[\begin{array}{c}
\left|i_{L}\right| \\
v_{0}
\end{array}\right]+\left[\begin{array}{c}
\frac{1}{L} \\
0
\end{array}\right]\left|v_{i n}\right|} \\
& {\left[\begin{array}{c}
\frac{d\left|i_{L}\right|}{d t} \\
\frac{d v_{0}}{d t}
\end{array}\right]=\left[\begin{array}{cc}
0 & -\frac{1}{L} \\
\frac{1}{C_{0}} & -\frac{1}{R_{L} C_{0}}
\end{array}\right]\left[\begin{array}{c}
\left|i_{L}\right| \\
v_{0}
\end{array}\right]+\left[\begin{array}{c}
\frac{1}{L} \\
0
\end{array}\right]\left|v_{i n}\right|}
\end{aligned}
$$

Kararlı durumda endüktans geriliminin ve kondansatör akımının ortalama değeri sıfırdır. Dönüştürücünün dinamik davranışı aşağıda gösterilmekte olan durum uzay ortalama matrisi tarafından belirlenmektedir.

$$
\left[\begin{array}{c}
\frac{d\left|i_{L}\right|}{d t} \\
\frac{d v_{0}}{d t}
\end{array}\right]=\left[\begin{array}{cc}
0 & -\frac{1-d}{L} \\
\frac{1-d}{C_{0}} & -\frac{1}{R_{L} C_{0}}
\end{array}\right]\left[\begin{array}{c}
\left|i_{L}\right| \\
v_{0}
\end{array}\right]+\left[\begin{array}{c}
\frac{d}{L} \\
0
\end{array}\right]\left|v_{i n}\right|
$$

Burada $T_{s}, L=L_{a}+L_{b}, d, C_{0}$ ve $R_{L}$ sırasıyla anahtarlama periyodunu, giriş endüktansını, anahtar doluluk oranını, çıkış kondansatörünü ve yükü ifade etmektedir. Matrislerdeki diferansiyel denklemleri çözmek için sayısal analiz yöntemlerinden aşağıda gösterilen kapal1-yamuk (implicit trapezoidal integration) kuralından yararlanılmaktadır. 


$$
x(n+1)=x(n)+\frac{h}{2}[f(x(n+1))+f(x(n))]
$$

Burada $h$ adım aralığıdır. Eşt.7'ye kapalı-yamuk kuralını uyguladığımızda sayısallaştırılmış endüktans akımı ve kondansatör gerilimi sırasıyla

$$
\begin{aligned}
& i_{L}(n+1)=i_{L}(n)+\frac{h}{2 L}\left[2 v_{i n}-(1-d)\left(v_{0}(n+1)+v_{0}(n)\right)\right] \\
& v_{0}(n+1)=v_{0}(n)+\frac{h}{2 C}\left[(1-d)\left(i_{L}(n+1)+i_{L}(n)\right)-\frac{1}{R_{L}}\left(v_{0}(n+1)+v_{0}(n)\right)\right]
\end{aligned}
$$

ifadeleri ile tanımlanmaktadır. Eşt.10 tekrar düzenlendiğinde çıkış yani kondansatör gerilimi şu biçimde olmaktadır:

$v_{0}(n+1)=\frac{1}{a_{1}}\left[v_{0}(n)+a_{2}\left(i_{L}(n+1)+i_{L}(n)\right)-a_{3} v_{0}(n)\right]$

Burada;

$a_{1}=1+\frac{h}{2 C R_{L}}, a_{2}=(1-d) \frac{h}{2 C}, a_{3}=\frac{h}{2 C R_{L}}$

Eşt. 11'i Eşt. 9'da yerine koyduğumuzda GFD devresinin giriş akımı yani endüktans akımı elde edilmektedir.

$i_{L}(n+1)=\frac{1}{1+a_{6}}\left[\left(1-a_{6}\right) i_{L}(n)+\left(a_{7}-a_{5}-a_{8}\right) v_{0}(n)+a_{4} v_{i n}\right]$

Burada;

$a_{4}=\frac{h}{L}, a_{5}=\frac{(1-d) h}{2 L a_{1}}, a_{6}=\frac{(1-d) h a_{2}}{2 L a_{1}}, a_{7}=\frac{(1-d) h a_{3}}{2 L a_{1}}, a_{8}=\frac{(1-d) h}{2 L}$

Sayısal denetleyicinin yapısı Şekil 5 'te gösterilmektedir. İşlemci ünitesi olarak yüksek hassasiyetli matematiksel işlemler yapabilen 32 bit TMS320F28335F DSP'si kullanılmıştır. Sayısal denetleyicinin girişleri giriş akımı, çıkış gerilimi ve giriş gerilimidir. Programda iki döngü vardır. İç döngü, giriş akımının şeklini denetlemekle sorumlu olmaktadır ve dış döngü ise PI (Oransal+İntegral) denetleyicisi yardımıyla çıkış geriliminin sabit olmasını sağlamaktadır. İç döngüde doğrultulmuş giriş akımı referans akımı ile karşılaştırılır, karşılaştırma sonucunda üretilen hata PI denetleyicisine aktarılır. PI denetleyicisinin çıkışında ise anahtarların doluluk oranı ayarlanmaktadır. 


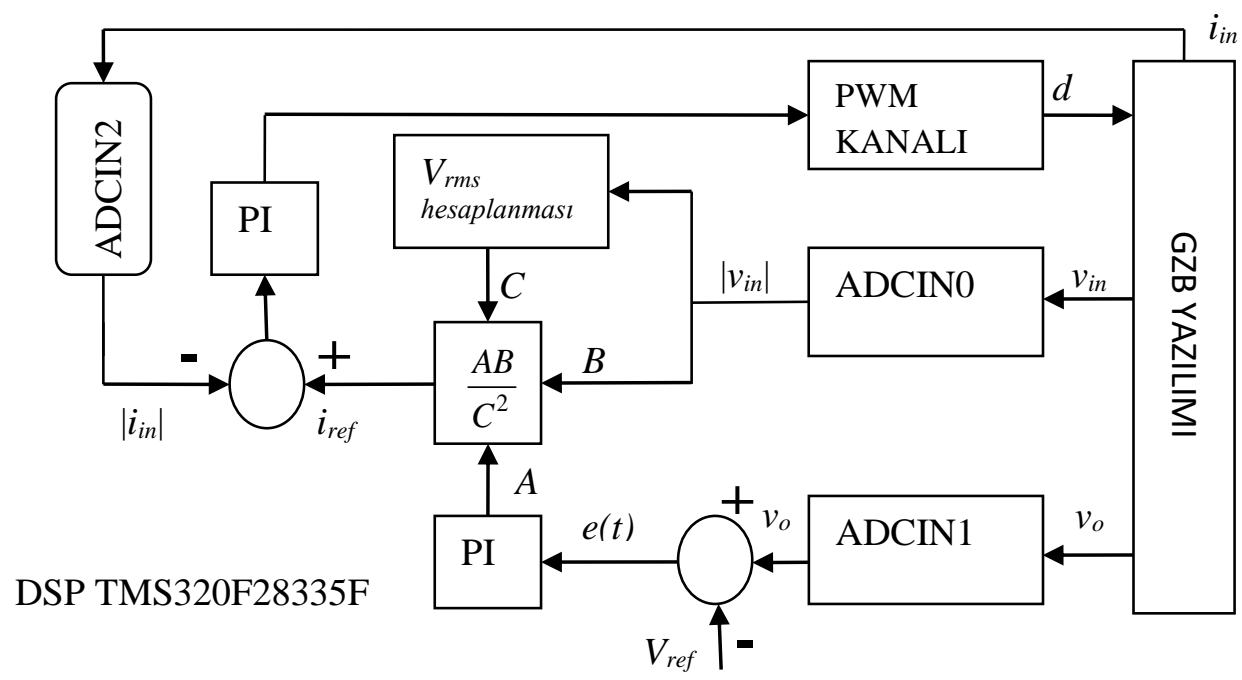

Şekil 5. Sayısal denetleyicinin yapısı

\section{BENZETIM SONUÇLARI}

Benzetim ve kapalı döngü donanım desteği çalışmalarında kullanılan değişkenler Tablo 1'de verilmiştir. İlk olarak, karşılaştırma yapabilmemiz için bu değişkenler kullanılarak MATLAB/Simulink programında benzetim çalışmaları yapılmış olup, sonuçlar Şekil 6 ve Şekil 7'de verilmiştir. Benzetim çalışmasında dönüştürücünün çalışmasını inceleyebilmek için çıkış gerilimi, giriş gerilimi ve giriş akımı gözlenmiştir. Şekil 6 tam yük ve Şekil 7 ise yük değişim benzetimini göstermektedir.

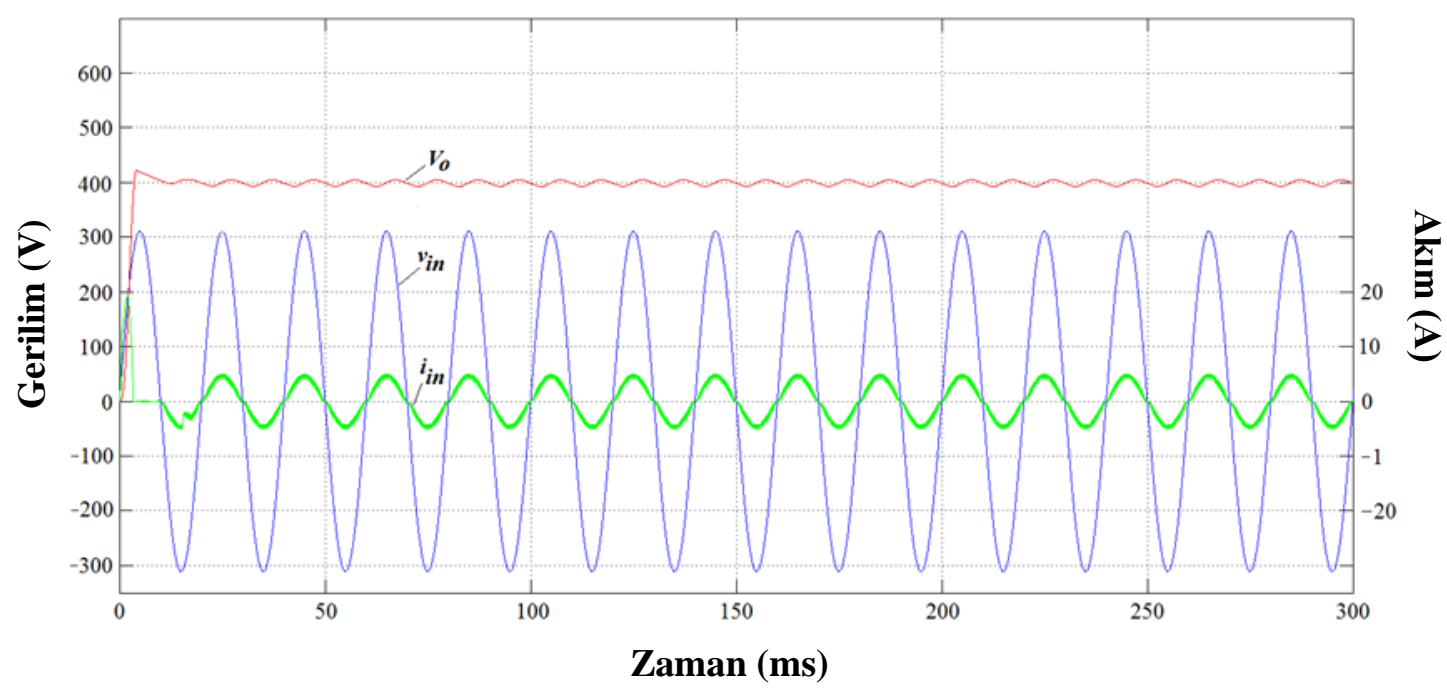

Şekil 6. Tam yükte çıkış gerilimi $\left(V_{o}\right)$, giriş gerilimim $\left(v_{\text {in }}\right)$ ve endüktans akımı $\left(i_{L}\right)$ 


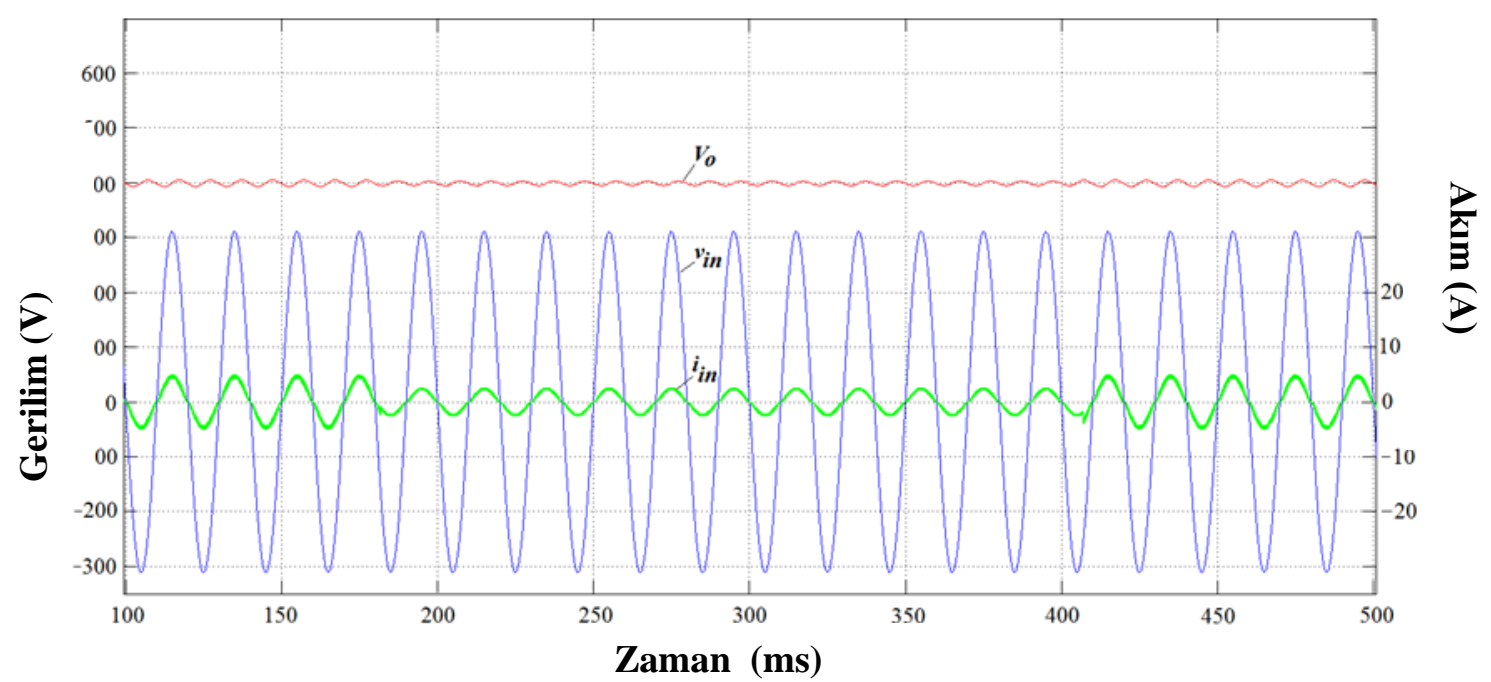

Şekil 7. Yük değişiminde çıkış gerilimi ( $\left.V_{o}\right)$, giriş gerilimim $\left(v_{\text {in }}\right)$ ve endüktans akımı $\left(i_{L}\right)$

Benzetim çalışmalarından Şekil 7 için benzetim için çalışma süresi 0.5 sn olmaktadır, ancak gerçekte işlemcinin harcadığı süre 15 sn olmaktadır. Dolayısıyla, daha uzun süreli benzetimlerde gömülü denetleyicinin geliştirilmesi ve test edilmesi için gereken süre daha da artabilmektedir. Kapalı döngü içerisinde benzetim için kullanılan sayısal denetleyicinin yapısı Şekil 5'te gösterilmektedir. Önerilen GFD devresinin matematiksel modeli çıkartılarak gerçek zamanlı bir sisteme gömülmüştür. Durum değişkenlerinin çözümünde kapalı-yamuk yöntemi kullanılmıştır ve açık kaynaklı işletim sistemi tarafından sistem yüksek öncelikli olarak çalıştırılmıştır. Dinamik belleğe erişim, sabit diske erişimden daha hızlı olmasından dolayı örnekleme adım aralı̆̆ azaltılabilmektedir. Bu nedenle, sistemin çalışma sırasında hesaplanan tüm veriler dairesel arabelleklere bölüştürülerek dinamik bellekte depolanmaktadır. Benzetim bittikten sonra bu veriler dinamik bellekten dosya sistemine kopyalanmaktadır. Şekil 8 ve Şekil 9, kapalı döngü içerisinde benzetiminde elde edilen dalga şekillerini göstermektedir. Kapalı döngü içerisinde benzetim için zaman aralığı $10 \mu$ s olarak ayarlanmıştır ve Şekil 9 için işlemci tarafindan harcanan süre 0.52 sn ölçülmüştür. Her iki benzetim çalışmalarından görüldüğü gibi genel davranışların yanı sıra anlık tepkilerin, gerçek zamanlı olmayan benzetim çalışmalarının tepkileriyle benzer olduğu görülmektedir.

Tablo 1. Benzetim ve kapalı döngü donanım desteği çalışmalarında kullanılan değişkenler

\begin{tabular}{ll}
\hline Bileşen & Değeri \\
\hline Giriş gerilimi $\left(v_{\text {in }}\right)$ ve şebeke & $220 \mathrm{~V}-50 \mathrm{~Hz}$ \\
frekansı $(f)$ & $500 \mathrm{~W}$ \\
Çıkış gücü $\left(P_{0}\right)$ & $400 \mathrm{~V}$ \\
Çıkış gerilimi $\left(V_{o}\right)$ & $10 \mu \mathrm{s}$ \\
Adım aralı̆̆ $(h)$ & $2 \mathrm{mH}$ \\
Giriş endüktansları $\left(L_{l}\right.$ ve $\left.L_{2}\right)$ & $470 \mu \mathrm{F}$ \\
Çıkıs kondansatörü & $50 \mathrm{kHz}$ \\
Anahtarlama frekansı $\left(f_{s}\right)$ & \\
\hline
\end{tabular}




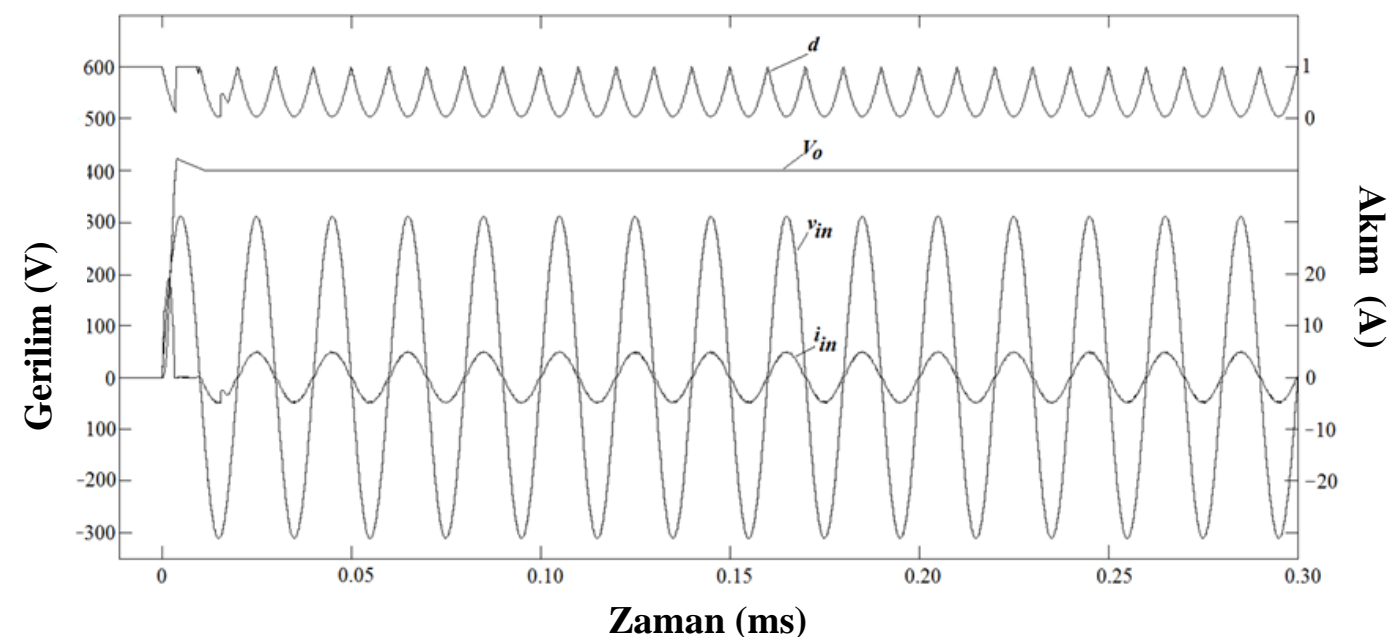

Şekil 8. Tam yükte kapalı döngü donanım desteği çalışmasının dalga şekilleri; anahtar doluluk oranı (d) çıkış gerilimi $\left(V_{o}\right)$, giriş gerilimim $\left(v_{i n}\right)$ ve endüktans akımı $\left(i_{L}\right)$

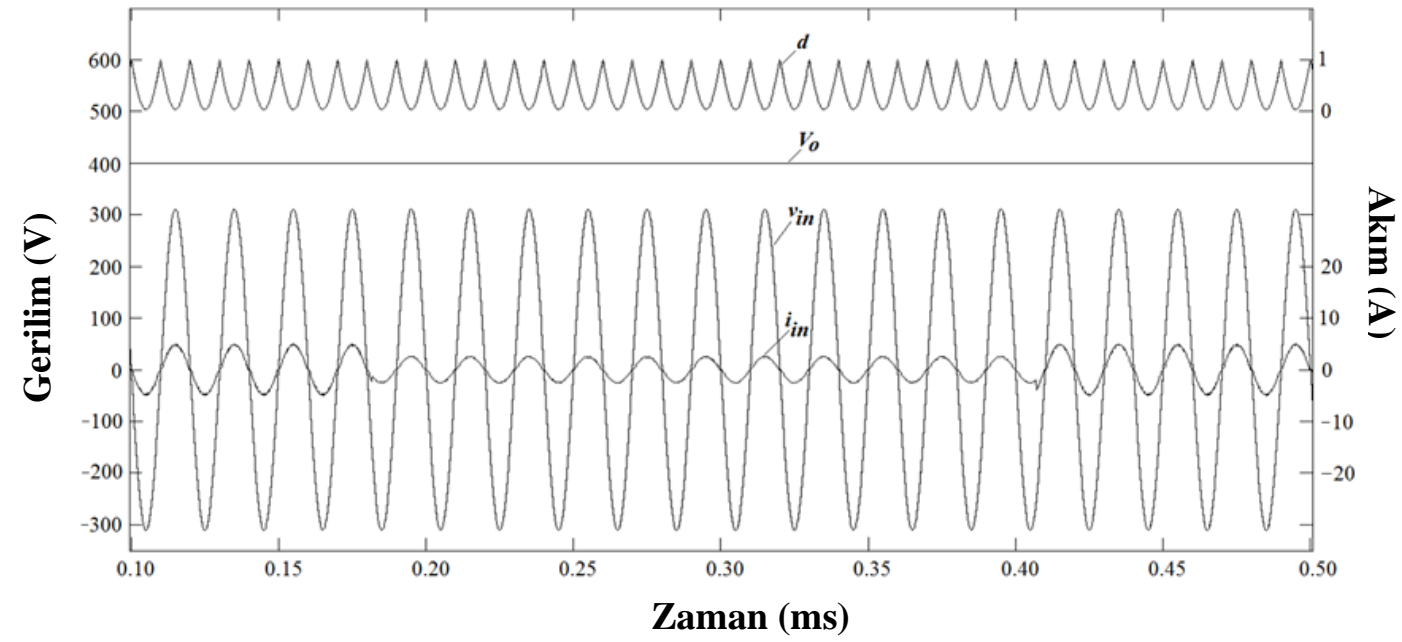

Şekil 9. Yük değişiminde kapalı döngü donanım desteği çalışmasının dalga şekilleri; anahtar doluluk oranı (d) çıkış gerilimi $\left(V_{o}\right)$, giriş gerilimim $\left(v_{i n}\right)$ ve endüktans akımı $\left(i_{L}\right)$

\section{SONUC}

$\mathrm{Bu}$ çalışmada, DSP denetimli güç dönüştürücülerinin geliştirilmesi ve test edilmesi için düşük maliyetli bir kapalı döngü donanım desteğinin benzetim çalışması sunulmuştur. Önerilen benzetim sonuçları, Simulink benzetim sonuçları ile karşılaştırılmış ve doğrulanmıştır. Bu benzetim altyapısına yeni matematiksel modeller eklenebilir ve örnekleme süresi daha da geliştirilebilir, böylece bu sistem daha karmaşık sistemlerin ayrıntılı analizinde kullanılabilmektedir. Bu şekilde, karmaşık sistemler için doğrulama test süresi azaltılabilmektedir. 


\section{KAYNAKLAR}

[1] C. Coopmans, M. Podhradský, N. V. Hoffer, "Software- and hardware-in-the-loop verification of flight dynamics model and flight control simulation of a fixed-wing unmanned aerial vehicle," Workshop on Research, Education and Development of Unmanned Aerial Systems (RED-UAS), Cancun, Meksika, 2015, ss. 115-122.

[2] D. Jung, P. Tsiotras, "Modeling and hardware-in-the-loop simulation for a small unmanned aerial vehicle", the AIAA Infotech Aerospace, CA, Birleşik Devletler, 2007, ss. 07-2768.

[3] M. Hu, G. Zeng, H. Yao, Y. Tang, "Processor-in-the-loop demonstration of coordination control algorithms for distributed spacecraft", Information and Automation (ICIA) 2010 IEEE International Conference on, Harbin, Çin, 2010, ss. 1008-1011.

[4] H. Zhang, Y. Zhang and C. Yin, "Hardware-in-the-Loop Simulation of Robust Mode Transition Control for a Series-Parallel Hybrid Electric Vehicle", IEEE Transactions on Vehicular Technology, c. 65 , s. 3, ss. 1059-1069, 2016.

[5] R. S. Kaarthik, P. Pillay, "Real-time power hardware-in-the-loop emulation of a parallel hybrid electric vehicle drive train", IEEE Transportation Electrification Conference (ITEC-India), Pune, Hindistan, 2017, ss. 1-6.

[6] A. Bouscayrol, "Different types of Hardware-In-the-Loop simulation for electric drives", IEEE International Symposium on Industrial Electronics, Cambridge, İngiltere, 2008, ss. 2146-2151.

[7] J. Jeon, J. Kim, H. Kim, S. Kim, C. Cho, J. Kim, J. Ahn, K. Nam, "Development of Hardware In-the-Loop Simulation System for Testing Operation and Control Functions of Microgrid", IEEE Transactions on Power Electronics, c.25, s.12, ss.2919-2929, 2010.

[8] L.Wei, G. Joos, J. Belanger,"Real-Time Simulation of a Wind Turbine Generator Coupled With a Battery Supercapacitor Energy Storage System", IEEE Transactions on Industrial Electronics, c.57, s.4, ss.1137-1145, 2010.

[9] M. Park, I. Yu , "A novel real-time simulation technique of photovoltaic generation systems using RTDS", IEEE Transactions on Energy Conversion, c.19, s.1, ss. 164- 169, 2004.

[10] Q. Cui, K. El-Arroudi, G. Joós, "Real-time hardware-in-the-loop simulation for islanding detection schemes in hybrid distributed generation systems", IET Generation, Transmission \& Distribution, c. 11, s. 12, ss. 3050-3056, 2017.

[11] J. Gamez García, J. Gómez Ortega, A. Sánchez García, S. Satorres Martínez, "Robotic software architecture for multisensor fusion system”, IEEE Trans. Ind. Electron., vol. 56, no. 3, pp. 766-777, Mar. 2009.

[12] N. R. Tavana, V. Dinavahi, "Real-time nonlinear magnetic equivalent circuit model of induction machine on FPGA for hardware-inthe-loop simulation", IEEE Trans. Energy Convers., c. 31, s. 2, ss. 520-530, 2016. 
[13] N. R. Tavana, V. Dinavahi, "A general framework for FPGA-based real-time emulation of electrical machines for HIL applications", IEEE Trans. Ind. Electron., c. 62, s. 4, ss. 2041-2053, 2015.

[14] G. Parma, V. Dinavahi, "Real-time digital hardware simulation of power electronics and drive", IEEE Trans. Power Del., c. 22, s. 2, ss. 1235-1246, 2007.

[15] A. Myaing, V. Dinavahi, "FPGA-Based Real-Time Emulation of Power Electronic Systems With Detailed Representation of Device Characteristics", Industrial Electronics IEEE Transactions on, c. 58, s.1, ss. 358-368, 2011.

[16] D. Zhang, H. Li, "Stochastic-based FPGA controller for an induction motor drive with integrated neural network algorithms", IEEE Trans. Ind. Electron., c. 55, s. 2, ss. 551-561, 2008.

[17] S. Lentijo, S. D'Arco, A. Monti, "Comparing the Dynamic Performances of Power Hardware-inthe-Loop Interfaces", IEEE Transactions on Industrial Electronics, c. 57, s. 4, ss. 1195-1207, 2010.

[18] B. Lu, X.Wu, H. Figueroa, A.Monti, "A low-cost real-time hardware in-the-loop testing approach of power electronics", IEEE Trans. Ind. Electron., c. 54, s. 2, s. 919-931, 2007.

[19] L. Huber, Y. Jang, M. M. Jovanovic, "Performance Evaluation of Bridgeless PFC Boost Rectifiers", IEEE Transactions on Power Electronics, c. 23, s. 3, s. 1381-1390, 2008. 